\title{
Correction: Alpha synuclein aggregation drives ferroptosis: an interplay of iron, calcium and lipid peroxidation
}

Plamena R. Angelova (D) - Minee L. Choi - Alexey V. Berezhnov • Mathew H. Horrocks - Craig D. Hughes • Suman De 1 - Margarida Rodrigues - Ratsuda Yapom • Daniel Little · Karamjit S. Dolt • Tilo Kunath Michael J. Devine (D) Paul Gissen (D) - Mikhail S. Shchepinov • Sergiy Sylantyev • Evgeny V. Pavlov • David Klenerman · Andrey Y. Abramov • Sonia Gandhi

Published online: 14 May 2020

(c) The Author(s), under exclusive licence to ADMC Associazione Differenziamento e Morte Cellulare 2020

Correction to: Cell Death \& Differentiation

https://doi.org/10.1038/s41418-020-0542-z
The Affiliation 'UCL Queen Square Institute of Neurology' appeared incorrectly in the original article as 'UCL Institute of Neurology'

This has been corrected in the PDF and HTML versions. 